\title{
Manipulating perception versus action in recalibration tasks
}

\author{
Christine J. Ziemer • Mia J. Branson • Benjamin J. Chihak • \\ Joseph K. Kearney • James F. Cremer • Jodie M. Plumert
}

Published online: 29 May 2013

(C) Psychonomic Society, Inc. 2013

\begin{abstract}
We conducted six experiments to examine how manipulating perception versus action affects perception-action recalibration in real and imagined blindfolded walking tasks. Participants first performed a distance estimation task (pretest) and then walked through an immersive virtual environment on a treadmill for $10 \mathrm{~min}$. Participants then repeated the distance estimation task (posttest), the results of which were compared with their pretest performance. In Experiments 1a, 2a, and 3a, participants walked at a normal speed during recalibration, but the rate of visual motion was either twice as fast or half as fast as the participants' walking speed. In Experiments $1 b, 2 b$, and $3 b$, the rate of visual motion was kept constant, but participants walked at either a faster or a slower speed. During pre- and posttest, we used either a blindfolded walking distance estimation task or an imagined walking distance estimation task. Additionally, participants performed the pretest and posttest distance estimation tasks in either the real environment or the virtual environment. With blindfolded walking as the distance estimation task for preand posttest, we found a recalibration effect when either the rate of visual motion or the walking speed was manipulated during the recalibration phase. With imagined walking as the distance estimation task, we found a recalibration effect when the rate of visual motion was manipulated, but not when the walking speed was manipulated in both the real environment and the virtual environment. Discussion focuses on how spatial-updating processes operate on perception and action and on representation and action.
\end{abstract}

\section{J. Ziemer ( $ه)$}

Missouri Western State University,

St. Joseph, MO, USA

e-mail: cziemer@missouriwestern.edu

B. J. Chihak

Aquinas College, Grand Rapids, MI, USA

M. J. Branson · J. K. Kearney · J. F. Cremer · J. M. Plumert

The University of Iowa, Iowa City, IA, USA
Keywords Perception and action · Navigation and spatial memory $\cdot$ Spatial cognition

As we locomote through the world, we constantly experience the relationship between our rate of physical movement and the rate of visual motion. For example, if we walk quickly down a sidewalk, we experience a faster rate of visual motion than if we walk slowly. Over time, this vast experience with the relationship between perception and action allows the system to build up expectations about how a given amount of movement will lead to a given amount of distance traveled. The regularity between the rate of movement and the distance traveled is altered only through mechanical devices such as moving sidewalks in airports. In such cases, a given rate of walking produces a greater amount of distance traveled than we normally experience. With enough experience on a moving sidewalk, people should recalibrate, or adapt to a new relationship between perception and action. In fact, several studies have shown that recalibration occurs even after only $10 \mathrm{~min}$ of experience with an altered relationship between perception and action (e.g., Mohler et al., 2004; Mohler et al., 2007; Proffitt, Stefanucci, Banton, \& Epstein, 2003; Rieser, Pick, Ashmead, \& Garing, 1995; Withagen, \& Michaels, 2002). Beyond these demonstrations of recalibration, however, relatively little is known about the processes that underlie the recalibration of perception and action. Here, we further examine how spatial-updating processes operate on perception and action and on representation and action.

\section{Coupling of perception, action, and representation}

According to Rieser and Pick (2007), perception, action, and representation are coupled together in an organized system that allows us to act adaptively whether perceiving (e.g., looking or listening) or representing (e.g., visualizing or imagining) the environment around us. Under normal 
circumstances, we use perception to guide action. Thus, if we want to pick up an object on the other side of a room, we simply look at the object while walking over to the location. This system of online control is highly accurate and efficient and likely governs much of our daily interactions with objects. Under other circumstances, however, we can use representation to guide action. For example, even without vision (due to blindness or darkness), we can walk over to pick up an object on the other side of a room (Rieser, Guth, \& Hill, 1986). This system of control relies on the coupling of action and representation, involving the coordination of motor actions with spatial knowledge.

Rieser and Pick (2007) hypothesized that similar processes operate on both perception-action coupling and representation-action coupling. In particular, when we walk with vision, we automatically keep track of the kinematics of our stepping (i.e., step rate and step length), relative to the rate of visual motion, to update our internal representation of where we are relative to the surrounding environment (Loomis, Da Silva, Fujita, \& Fukusima, 1992). This process is referred to as spatial updating. Rieser et al. (1995) posited that the coupling of perception and action can account for the coupling of representation and action. In other words, as we learn the relationship between our actions and our perception under normal conditions (e.g., with visual feedback), we are able to map this experience to our representation of space in order to act adaptively when conditions require us to rely on representation. Specifically, when people walk without vision, spatial-updating processes operate on representations of the surrounding environment, allowing people to know where they are in relation to the surrounding environment. As a result, they are able to walk without vision to targets up to $30 \mathrm{~m}$ away fairly accurately (Farrell \& Thomson, 1998, 1999).

When the normal link between rate of physical movement and visual movement is altered (as in the moving sidewalk example), spatial-updating processes cause people to recalibrate the link between perception and action. We can measure this change in the perception-action-representation relationship in tasks where people rely on their representation of the environment while performing an action task without vision. Tasks such as blindfolded walking or blindfolded imagined walking to previously seen targets have been used to probe changes in the perception-actionrepresentation system (e.g., Kunz, Creem-Regehr, \& Thompson, 2009; Mohler et al., 2004; Mohler et al., 2007; Rieser et al., 1995). In these types of tasks, participants rely on their representation of space coupled with their recalibrated sense of how much action it takes to reach the target. Participants overshoot the target if they have experienced more walking in order to cover a given distance, and they undershoot the target if they have experienced less walking to cover a given distance.

\section{Manipulating the rate of visual motion to produce recalibration of perception and action}

Several researchers have demonstrated perception-action recalibration in laboratory settings (e.g., Mohler et al., 2004; Mohler et al., 2007; Proffitt et al., 2003; Rieser et al., 1995; Withagen, \& Michaels, 2002). The perceptionaction relationship is usually manipulated by altering the rate of the environmental visual motion (i.e., faster or slower) in relation to the participant's walking speed. A common task used to measure change in the perceptionaction-representation relationship is blindfolded walking, in which participants attempt to walk blindfolded to previously viewed targets both before and after the recalibration experience. Blindfolded walking has been shown to be a reliable way to measure participants' representation of distance (Farrell \& Thomson, 1998, 1999; Rieser, Ashmead, Talor, \& Youngquist, 1990).

In the original recalibration study, Rieser et al. (1995) found that people recalibrated the link between perception and action after a brief period of altered covariation of biomechanical activity and visual motion. Participants completed a pre- and posttest in which they viewed targets at $8 \mathrm{~m}$ away and then attempted to walk to them with their eyes closed. Between the pre- and posttest, participants experienced a "rearrangement," or adaptation, phase in which they walked at a constant speed on a motor-driven treadmill that was being pulled by a tractor at a faster or slower speed. Participants therefore experienced visual motion at either a faster or slower rate than the biomechanical speed at which they were walking. Using a blindfolded walking task, Rieser et al. (1995) found that participants who experienced slower visual motion walked past the target during the posttest, whereas participants who experienced faster visual motion stopped short of the target during posttest. These results indicate that the participants learned the new relationship between walking speed and perceived distance traveled during the adaptation period. When the visual motion was fast relative to their speed of walking, participants learned that they traveled more distance with each step than before, and when the visual motion was slow relative to their speed of walking, they learned that they traveled less distance with each step.

Other researchers have replicated Rieser et al.'s (1995) recalibration effect, using a treadmill in a virtual environment during the adaptation phase (e.g., Mohler et al., 2004; Mohler et al., 2007; Proffitt et al., 2003; Withagen \& Michaels, 2002). The use of a virtual environment is helpful for eliminating potential methodological problems with Rieser et al.'s (1995) study, such as a raised eye height and the view of the tractor while being pulled on a treadmill. In Mohler et al. (2004, 2007), participants first performed a pretest in a real hallway, which involved nine trials of 
walking blindfolded to targets at different distances $(6,8$, and $10 \mathrm{~m})$. Following the pretest, participants walked on a treadmill in a virtual environment that was modeled after the real hallway. During the adaptation phase in the virtual environment, visual motion was adjusted to be faster than, slower than, or the same as the speed of walking. Following the adaptation experience, participants performed a posttest that was identical to the pretest. They found that participants who had experienced slower visual motion in the virtual environment overshot the target distances during posttest, relative to pretest, by $11 \%$, while participants who had experienced faster visual motion undershot the same distances at posttest, relative to pretest, by $6 \%$. It is important to note that the recalibration rates found in these studies are not comparable in size to the relationship established during the adaptation experience. Although the rate of visual motion may be twice as fast or half as fast as participants' walking speed during adaptation, the recalibration effects observed during posttest are much smaller.

To date, research on perception-action recalibration has attempted to produce recalibration only by changing the rate of visual motion during adaptation, while holding walking speed constant. No studies have examined whether recalibration occurs to the same extent when the perception-action relationship is altered by changing the speed of physical movement while keeping the rate of visual motion constant. In order to better understand the processes underlying perception-action recalibration, it is important to determine whether we can experimentally produce recalibration through manipulating different components of the perception-action system. If people are sensitive to the relationship between physical movement and visual motion, changing this relationship either through manipulating physical movement (while keeping visual motion constant) or through manipulating visual motion (while keeping physical movement constant) should produce recalibration. Alternatively, if people are sensitive only to the rate of visual motion, changing the relationship by manipulating physical movement (while keeping visual motion constant) should not produce recalibration.

\section{Recalibration of perception and action using blindfolded walking and imagined walking tasks}

As was discussed above, recalibration has typically been studied via a blindfolded walking task during pre- and posttest. This task is considered the gold standard for studying recalibration of perception and action, since it allows for a fairly direct test of whether people's sense of the amount of movement required to travel to a previously seen target has changed based on a recalibration experience. Presumably, blindfolded walking engages spatial-updating processes based on the tight coupling between perception, action, and representation.

More recently, imagined walking has been used to assess distance perception in both real and virtual environments (Decety, Jeannerod, \& Prablanc, 1989; Grechkin, Nguyen, Plumert, Cremer, \& Kearney, 2010; Kunz et al., 2009; Plumert, Kearney, Cremer, \& Recker, 2005; Ziemer, Plumert, Cremer, \& Kearney, 2009). The basic task is identical to blindfolded walking to targets, except that participants imagine walking to targets while standing in place. Kunz et al. recently tested recalibration of perception and action using this imagined walking task. Participants walked on a treadmill through a large-screen virtual environment during adaptation,but made imagined walking distance estimates during pre- and posttest. Participants started a stopwatch when they imagined themselves beginning to walk to the target and stopped it when they imagined reaching the target (without ever looking at the stopwatch). When estimating the amount of time it would take them to walk to targets at posttest, relative to pretest, participants in the slower visual motion condition overshot the target, relative to pretest, by $15 \%$, and participants in the faster visual motion condition undershot the target, relative to pretest, by $14 \%$. Kunz et al. concluded that recalibration is similar when tested with an imagined walking task, as compared with the standard blindfolded walking task.

The Kunz et al. (2009) study clearly indicated that manipulating visual motion during adaptation (while holding walking speed constant) leads to systematic overshooting and undershooting at test in an imagined walking task. In fact, the recalibration effect appears to be about twice as large as in a blindfolded walking task. They concluded that imagined movement relies on motor simulation processes, suggesting a functional equivalence between real walking and imagined walking. At present, however, it is not known whether manipulating walking speed during adaptation (while holding rate of visual motion constant) also leads to systematic overshooting and undershooting in an imagined walking task. If the same spatial-updating processes operate on representation and action (whether real or simulated action), we should expect to see the same pattern of effects across blindfolded walking and imagined walking.

\section{Recalibration of perception and action in real and virtual environments}

One final element that might influence the extent to which people exhibit recalibration of perception and action is the environment in which the pre- and posttest tasks take place. We have vast amounts of experience with the relationship between our walking speed and distance traveled in the real world. Given this, recalibration studies to date have tested 
perception-action recalibration by having people estimate distances in the real world before and after the recalibration experience (even when the recalibration experience occurs in a virtual environment). At present, however, little is known about how recalibration experiences affect perception and action in virtual environments.

Many studies have found that people significantly underestimate distances in virtual environments, suggesting that people may be more malleable in virtual environments (Nguyen et al., 2011; Swan, Jones, Kolstad, Livingston, \& Smallman, 2007; Thompson et al., 2004; Witmer \& Kline, 1998; Witmer \& Sadowski, 1998). In short, people do not have a very good sense of how much movement is required to travel a given distance in a virtual environment; they systematically underestimate the amount of movement required to reach the desired destination. One possible explanation for this may be that virtual environments appear compressed. Research has shown that giving participants time to interact and become familiar with the virtual environment greatly increases their accuracy in distance estimation tasks (Interrante, Anderson, \& Ries, 2006; Waller \& Richardson, 2008). Due to the relative unfamiliarity of the virtual environment used in recalibration studies and the fact that these environments are more cue impoverished, participants may rely more heavily on the perception-action relationship learned during the recalibration phase. Therefore, one might expect a stronger recalibration effect when testing participants in a virtual environment, as compared with the real environment.

\section{The present investigation}

The goal of the present investigation was to better understand the spatial-updating processes that operate on perception and action and on representation and action. With regard to spatial-updating processes operating on perception and action, we wanted to test whether the recalibration effect depends on the ratio of visual motion to walking speed. With regard to the spatial-updating processes that operate on representation and action, we were interested in whether manipulations of either perception or action during adaptation extend to both blindfolded walking and imagined walking. We examined both of these issues by directly comparing the amount of recalibration in blindfolded walking and imagined walking tasks when the perception-action link was manipulated via the rate of visual motion (while keeping walking speed constant) or walking speed (while keeping rate of visual motion constant). Finally, we examined whether the type of environment in which participants make their pre- and posttest distance estimates affects the recalibration of perception and action by comparing imagined walking estimates in real and virtual environments.

\section{Experiments 1a and 1b}

The goal of Experiments 1a and $1 \mathrm{~b}$ was to determine whether people would recalibrate perception and action in the standard blindfolded walking task when we manipulated either the rate of visual motion (while keeping walking speed constant) or the walking speed (while keeping the rate of visual motion constant). In Experiment 1a, we had participants walk on a treadmill in an immersive virtual environment, with the rate of visual motion either twice as fast or half as fast as their walking speed. As in previous work, we expected participants in the fast visual motion condition to undershoot distances at posttest, relative to pretest, and participants in the slow visual motion condition to overshoot distances at posttest, relative to pretest. In Experiment 1b, we had participants walk on the treadmill at either a fast or a slow walking speed during adaptation while keeping the rate of visual motion the same between conditions. If recalibration occurred, participants in the slow-walking condition of Experiment $1 \mathrm{~b}$ should undershoot distance at posttest, relative to pretest (since during adaptation, it took them less physical movement to cover a given visual distance), and participants in the fast-walking condition should overshoot distance at posttest, relative to pretest (since during adaptation, it took them more physical movement to cover a given visual distance).

\section{Experiment 1a: Method}

\section{Participants}

Twenty-three adults (9 females) participated. Participants were recruited from an introductory psychology course at the University of Iowa and received course credit for their participation.

\section{Apparatus and materials}

The virtual environment was a repeating section of hallway modeled after the actual hallway used in pre- and posttest. The virtual environment was displayed on three 10 -ft-wide $\times$ 8 -ft-high screens placed at right angles relative to one another, forming a three-walled room. To accommodate the height of the treadmill, the floor on which participants stood was $1 \mathrm{ft}$ above the bottom of the vertical screens, so the effective screen height was $7 \mathrm{ft}$. Participants stood on the treadmill approximately $5 \mathrm{ft}$ from the front screen, midway between the side screens. Three Projection Design F1+ projectors were used to rear project high-resolution graphics $(1,280 \times 1,024$ pixels) onto the screens, providing participants with approximately (depending on the participant's height) $224^{\circ}$ horizontal and $46^{\circ}$ vertical field of vision of nonstereoscopic, immersive visual imagery. The viewpoint of the scene was adjusted for each participant's eye height. 
The treadmill was a Woodway Wide Path motorized treadmill. The belt of the treadmill was $21.5 \mathrm{in}$. wide and 45 in. long. While walking on the treadmill, participants wore a safety harness (Safewaze Apache or Petzl 8003 Full Body harness), which was attached to the ceiling to prevent them from falling. Participants wore a Mindfold blindfold and headphones playing white noise while making blindfolded walking distance estimates during the pre- and posttest phases. The target consisted of a green circle (13 in. in diameter, $0.25 \mathrm{in}$. thick) placed on the floor, with the center of the circle at the target distance for each trial. Participants' distance estimations were measured using a Bosch digital laser finder (model DLR165K).

\section{Design and procedure}

Distance estimation pretest Participants were first allowed to practice walking blindfolded to the end of the hallway and back (hallway length was $25.3 \mathrm{~m}$ ). Participants then performed a blindfolded walking distance estimation pretest. This task was completed in a hallway directly outside of the virtual environment lab. For each trial, participants viewed a target on the floor for about $5 \mathrm{~s}$. Participants were instructed to form a good mental image of the target and the surrounding environment while viewing the target. They were informed that an experimenter would move the target out of the way and that they were to stop when they thought they were in the exact spot the target had been. Once they felt they had formed a good mental image, they pulled down the blindfold and attempted to walk to the target. If participants veered too far to the left or right while walking blindfolded, the experimenter would tap them gently on the arm to correct their path, to prevent them from making contact with the hallway wall. The pretest began with two practice trials with targets located at 7 and $9 \mathrm{~m}$. Participants did not wear the white noise headphones during the two practice trials to allow the experimenters to give directions and the participants to ask questions. After the practice trials, participants wore noise-canceling headphones playing white noise for the remainder of the pretest and posttest. During the test trials, targets were located at 6,8 , and $10 \mathrm{~m}$. Participants saw each distance 3 times, randomly ordered in blocks of three (nine trials total). After each trial, the experimenter led the participant back to the starting position while still blindfolded. Participants received no feedback about their estimates during practice or test trials.

Adaptation The adaptation phase began immediately after the pretest. Participants were taken into the lab, outfitted with the safety harness, and asked to step onto the treadmill (Fig. 1). Before the displays were turned on, participants were allowed to walk on the treadmill for approximately $1 \mathrm{~min}$ in order to familiarize them with walking on the

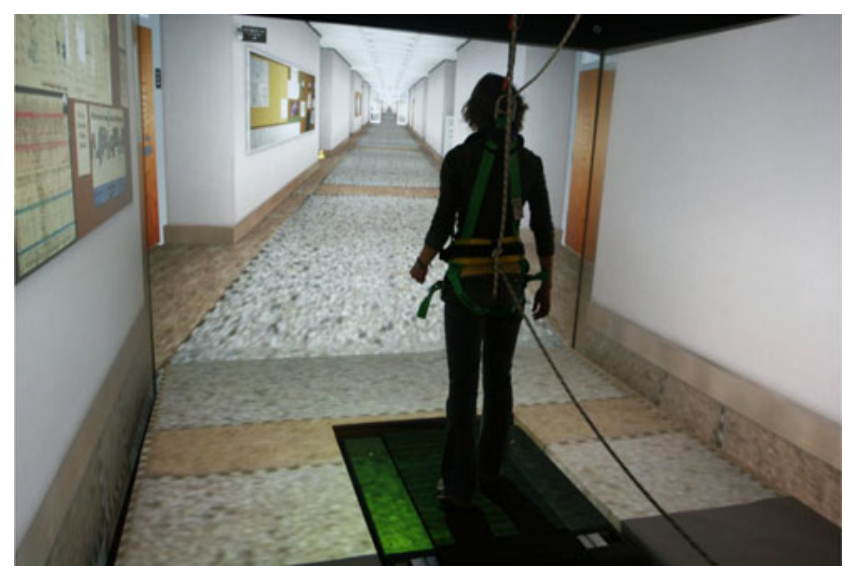

Fig. 1 Large-screen immersive virtual environment setup with treadmill and harness

treadmill and to select a comfortable walking speed. All participants started at $3 \mathrm{mph}$ speed and then told the experimenter whether they wanted to walk faster or slower. The experimenter then adjusted the walking speed up or down until the participant felt comfortable. The mean walking speed was $2.89 \mathrm{mph},(S D=0.198)$. Participants then walked through an immersive virtual environment on the treadmill for $10 \mathrm{~min}$ at the walking speed they had selected. Using a between-subjects design, participants were randomly assigned to either the fast visual motion condition or the slow visual motion condition. Specifically, the interface was configured such that the rate of visual motion was either half as fast (slow visual motion condition) or twice as fast (fast visual motion condition) as the participant's walking speed. The virtual environment was a model of the same hallway in which the pre- and posttest were conducted (Fig. 2). Participants were told to look for ducks and bunnies hidden in the doorways of the hallway while walking through the virtual environment and to report when they saw one. The search for ducks and bunnies was designed to encourage participants to look from side to side while walking to enhance their perception of lamellar flow (Durgin et al., 2005).

Distance estimation posttest Immediately following adaptation, participants completed the blindfolded walking distance estimation posttest. Participants were blindfolded and led off of the treadmill and back out to the hallway. Participants then repeated the same blindfolded walking distance estimation task as that used in the pretest. The target distances were 6,8 , and $10 \mathrm{~m}$ presented in three blocks of three in a new random order.

\section{Measures}

Scores represented the percentage of distance walked at posttest relative to pretest. Posttest means for each distance were divided by pretest means for each distance $(6,8$, and 
Fig. 2 Virtual environment hallway (left) and real hallway (right)
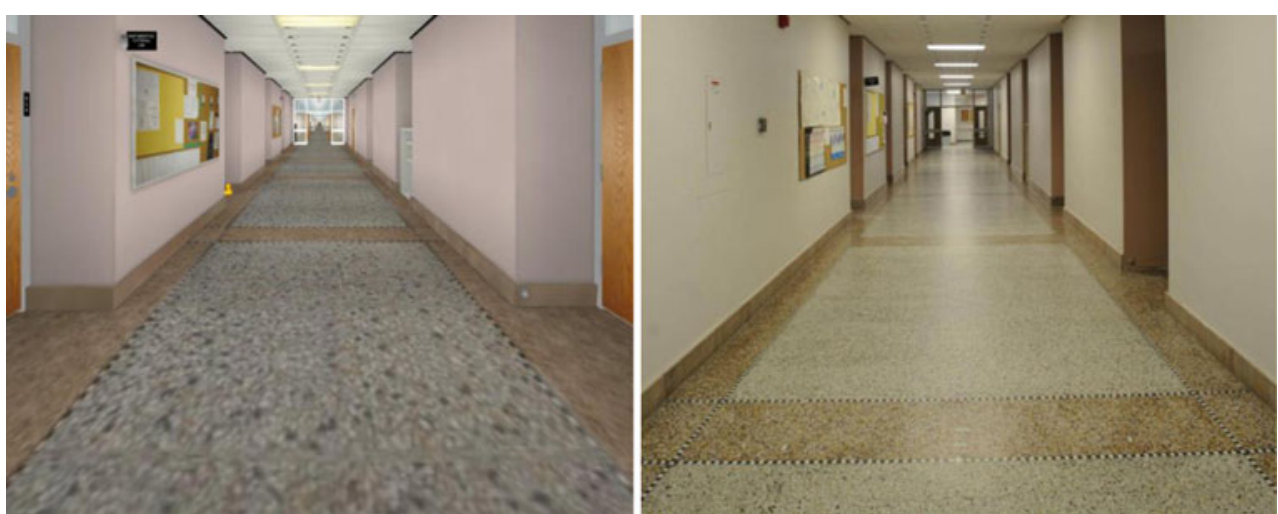

$10 \mathrm{~m})$ to create a percentage score for each distance. This measure allowed us to look at how the recalibration experience affected posttest distance estimates relative to pretest distance estimates. Three participants with a score 1.5 standard deviations above or below the mean (averaged across distances) were excluded from the analyses ( 2 from the fast visual motion condition and 1 from the slow visual motion condition).

\section{Experiment 1a: Results and discussion}

We first examined the extent to which participants undershot or overshot the actual distances prior to any recalibration experience. Participants' mean pretest distance estimation scores were $5.49 \mathrm{~m}(S D=0.79)$ for the 6-m distance, $7.68 \mathrm{~m}$ $(S D=0.99)$ for the $8-\mathrm{m}$ distance, and $10.54 \mathrm{~m}(S D=1.34)$ for the 10-m distance. One-sample $t$-tests comparing each mean with the actual distance revealed that only the 6-m pretest estimate differed significantly from the actual distance, $t(19)=-2.89, p<.01$.

The main goal of Experiment 1a was to replicate the standard recalibration effect in a blindfolded walking task. If recalibration is successful, we would expect that the percentage of distance walked at posttest, relative to pretest, would be significantly larger in the slow visual motion condition than in the fast visual motion condition. Percentage scores were entered into a condition (fast visual motion vs. slow visual motion $) \times$ distance $(6$ vs. 8 vs. $10 \mathrm{~m})$ mixed model ANOVA with the first factor as a betweensubjects variable and the second factor as a within-subjects variable. As was expected, we found a significant effect of condition, $F(1,18)=5.25, p<.05, \eta_{\mathrm{p}}{ }^{2}=.23$, with the mean for the fast visual motion group $(M=98.53 \%, S D=9.13)$ significantly lower than the mean for the slow visual motion group $(M=106.54 \%, S D=5.65$; Fig. 3$)$. There was also a significant effect of distance, $F(1,18)=4.65, p<.05, \eta_{\mathrm{p}}{ }^{2}=$ .21 , but no significant condition $\times$ distance interaction, $F(1,18)=0.19$, n.s. Mean percentage scores were $105.62 \%$ $(S D=12.25)$ for the 6-m distance, $102.33 \%(S D=8.82)$ for the $8-\mathrm{m}$ distance, and $98.45 \%(S D=11.25)$ for the $10-\mathrm{m}$ distance. Percentage scores for the 6- and 10-m distances differed significantly but did not differ significantly for the 8- and 10-m distances or for the 6- and 8-m distances.

We also directly compared the distance walked during pretest and posttest in the fast and slow visual motion conditions. Paired samples $t$-tests comparing the average distance walked at pretest to the average distance walked at posttest showed a significant difference between pre- and posttest for the slow condition $(M=7.65 \mathrm{~m}, S D=0.84$ for pretest, $M=8.10 \mathrm{~m}, S D=0.92$ for posttest), $t(8)=-3.189$, $p<.05, d=0.58$, but no significant difference between preand posttest for the fast condition $(M=8.11 \mathrm{~m}, S D=1.09$ for pretest; $M=7.89 \mathrm{~m}, S D=1.25$ for posttest), $t(10)=$ $0.943, p=.368$.

The significant condition effect indicates that participants recalibrated the link between perception and action when the rate of visual motion was manipulated during adaptation and the walking speed was held constant. Although the recalibration effect was not large given the amount of increase or decrease in the rate of visual motion, the results of

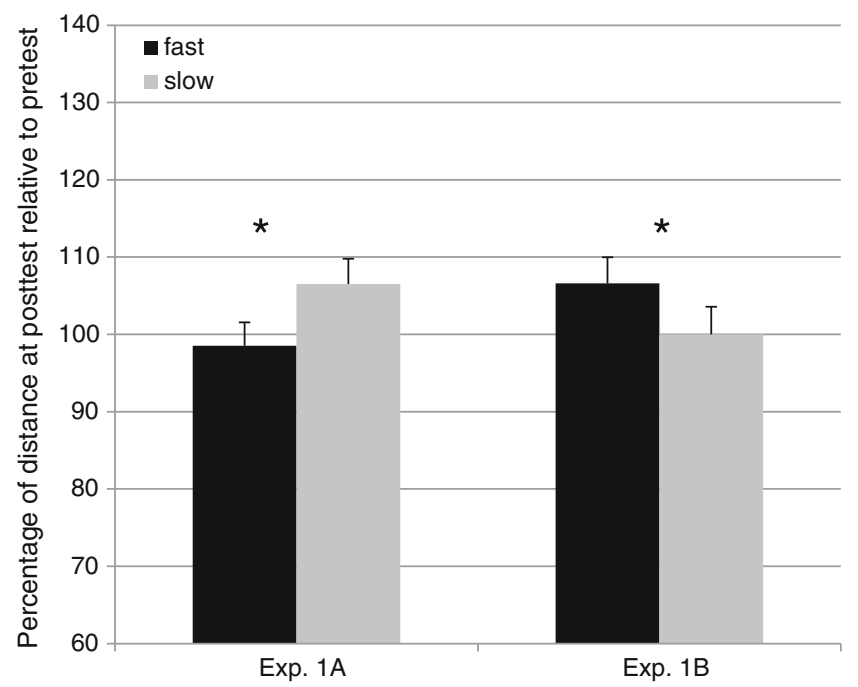

Fig. 3 Percentage of distance estimated at posttest, relative to pretest, in fast and slow conditions for Experiments $1 \mathrm{a}$ and $1 \mathrm{~b}$ 
this experiment are consistent with the effects of previous recalibration studies that manipulated the rate of visual motion to produce recalibration (Mohler et al., 2004; Mohler et al., 2007; Rieser et al., 1995). The goal of Experiment $1 \mathrm{~b}$ was to examine whether participants also recalibrate perception and action when the walking speed is manipulated during adaptation and the rate of visual motion is held constant. We also videotaped participants' blindfolded walking distance judgments during both preand posttest in order to determine whether walking speeds and walking times at posttest, relative to pretest, differed across conditions.

\section{Experiment 1b: Method}

\section{Participants}

Thirty-five adults (18 females) participated. Participants were recruited in the same manner as in the previous experiment. ${ }^{1}$

\section{Apparatus and materials}

The apparatus and materials were the same as those used in Experiment 1a. For this and the remaining experiments, a Sanyo PLC-WXE45 projector was used to project the ground surface around the treadmill onto the floor. We also used a video camera mounted on a tripod to videotape participants' pre- and posttest blindfolded walking.

\section{Design and procedure}

All aspects of the pretest and posttest procedure were the same as in Experiment 1a. During adaptation, participants were assigned to either a fast $(3.5 \mathrm{mph})$ or slow $(1.5 \mathrm{mph})$ treadmill walking speed during adaptation. The rate of visual motion for both conditions was the same $(2.5 \mathrm{mph}){ }^{2}$

\section{Measures}

Scores represented the percentage of the time estimated at posttest, relative to pretest. Five participants with a score 1.5 standard deviations above or below the mean (averaged across distances) were excluded from the analyses (3 from the fast walking speed condition and 2 from the slow walking speed condition).

\footnotetext{
${ }^{1}$ All of the participants were new in each of the six experiments presented here.

${ }^{2}$ We were unable to use the same 1:2 rate of visual motion to walking speed as we used in the first set of experiments because we were constrained by how fast we could make participants walk on the treadmill before their gait would change to a run.
}

Video recordings of participants' blindfolded walking during the pretest and posttest were coded for the time elapsed from start to stop. Intercoder reliability $(N=5)$ was $r=.967$ for time to walk to the target. We used the time and distance participants walked to calculate their walking speed during pre- and posttest.

\section{Experiment 1b: Results and discussion}

We again first examined participants' pretest distance estimates relative to the actual target distances. Mean estimated pretest distances were $5.05 \mathrm{~m}(S D=0.69)$ for the $6-\mathrm{m}$ distance, $7.30 \mathrm{~m}(S D=1.0)$ for the $8-\mathrm{m}$ distance, and $10.01 \mathrm{~m}(S D=1.54)$ for the $10-\mathrm{m}$ distance. One-sample $t$-tests showed that the 6-m, $t(29)=-7.462, p<.001$, and $8-\mathrm{m}, t(29)=-3.82, p<.01$, pretest estimates differed significantly from the actual distances, but not the $10-\mathrm{m}$ pretest estimates.

The goal of Experiment $1 \mathrm{~b}$ was to examine whether the recalibration effect could be achieved by manipulating action (walking speed) instead of perception (rate of visual motion). A condition (2) $\times$ distance (3) mixed model ANOVA on percentage scores revealed a significant effect of condition, $F(1,28)=6.63, p<.05, \eta_{\mathrm{p}}{ }^{2}=.19$, indicating that the mean for the fast visual motion condition $(M=$ $106.6 \%, S D=6.74)$ was significantly higher than that for the slow visual motion condition $(M=99.99 \%, S D=7.32$; Fig. 3). There was also a significant effect of distance, $F(1,28)=8.40, p<.01, \eta_{\mathrm{p}}{ }^{2}=.23$, but no significant distance $\times$ condition interaction, $F(1,28)=0.24$, n.s. Mean percentage scores were $106.10 \%(S D=7.1)$ for the 6-m distance, $102.52 \%(S D=10.31)$ for the $8-\mathrm{m}$ distance, and $101.26 \%(S D=9.87)$ for the $10-\mathrm{m}$ distance. Percentage scores differed significantly between the 6- and the 8- and 10-m distances, but not between the 8- and 10-m distances.

We also directly compared the distance walked during pre-test and posttest in the fast- and slow-walking conditions. Paired samples $t$-tests comparing the average distance estimated at pretest with the average distance estimated at posttest showed a significant difference between pre- and posttest for the fast condition $(M=7.32 \mathrm{~m}, S D=0.80$ for pretest; $M=7.71 \mathrm{~m}, S D=0.66$ for posttest), $t(14)=-3.004$, $p<.01, d=0.72$, but no significant difference between preand posttest for the slow condition $(M=7.59 \mathrm{~m}, S D=1.2$ for pretest; $M=7.49 \mathrm{~m}, S D=0.88$ for posttest), $t(14)=$ $0.629, p=.539$, n.s.

Using the videotapes of participants' pre- and posttest performance, we also analyzed participants' walking times and walking speeds. A one-way ANOVA on the mean percentage of walking time at posttest, relative to pretest, revealed no significant difference between the two conditions, $F(1,28)=0.933$, n.s. However, the mean percentage of walking speed at posttest, relative to pretest, was 
significantly higher in the fast-walking condition $(M=$ $114.28 \%, S D=13.22)$ than in the slow-walking condition $(M=102.42 \%, S D=8.57), F(1,28)=8.50, p<.01, \eta_{\mathrm{p}}{ }^{2}=$ .19. As a result, participants in the fast walking speed condition walked further than did participants in the slow walking speed condition. ${ }^{3}$

Together, Experiments $1 \mathrm{a}$ and $1 \mathrm{~b}$ demonstrate that recalibration occurs regardless of whether we manipulate perception or action (although the effect was stronger for overshooting than for undershooting at posttest, relative to pretest). Since both perception and action are crucial for recalibration, manipulating either component should result in a change in the perception-action relationship, measurable through changes in the distance walked at posttest relative to pretest.

\section{Experiments 2a and 2b}

One question the results of Experiment $1 \mathrm{a}$ and $1 \mathrm{~b}$ raise is whether these effects extend to imagined walking distance estimates. In Experiment 2a, we manipulated the rate of visual motion during the adaptation phase and used an imagined walking distance estimation task at pre- and posttest. In Experiment 2b, we manipulated the walking speed during the adaptation phase and again used an imagined walking distance estimation task at pre- and posttest.

\section{Experiment 2a: Method}

\section{Participants}

Thirty adults (12 females) participated. Participants were recruited in the same manner as in the previous experiments.

\section{Apparatus and materials}

The apparatus and materials were identical to those used in Experiments $1 \mathrm{a}$ and $1 \mathrm{~b}$. Additionally, participants used a handheld, digital stopwatch to indicate the time they imagined it would take them to walk to the targets.

\section{Design and procedure}

Participants first performed an imagined walking distance estimation pretest. This task was completed in a hallway directly outside of the virtual environment lab. We first obtained an estimated walking speed (sans blindfold) for

\footnotetext{
$\overline{3}$ Although we did not videotape participants' performance in Experiment 1a, there is no reason to believe that participants in the fast and slow visual motion conditions walked at different speeds at posttest.
}

participants by having them walk the length of the hallway $(25.3 \mathrm{~m})$ two times. We took the average of these two trials to compute each participant's estimated walking speed. For each trial during the distance estimation task, participants viewed a target on the floor for $5 \mathrm{~s}$ and then pulled down the blindfold and imagined walking to the target location. Participants were instructed to form a good mental representation of the target and the surrounding environment while viewing the target. Participants started the stopwatch when they imagined starting to walk to the target and stopped the stopwatch when they imagined reaching the target. Participants stood in place while making imagined walking distance estimations. As in the previous experiments, the pretest began with two practice trials with targets located at 7 and $9 \mathrm{~m}$ and was followed by nine test trials with targets located at 6,8 , and $10 \mathrm{~m}$. After each trial, the experimenter took the stopwatch from the participant and recorded the time elapsed. Participants were not allowed to see their times and received no feedback about their estimates during practice or test trials.

The adaptation phase began immediately after the pretest and followed the same procedure as in Experiment 1a. Immediately following adaptation, participants completed an imagined walking distance estimation posttest that was identical to the pretest.

\section{Measures}

Scores represented the percentage of the time estimated at posttest relative to pretest. Three participants with a score 1.5 standard deviations above or below the mean (averaged across distances) were excluded from the analyses (1 from the fast visual motion condition and 2 from the slow visual motion condition).

Experiment 2a: Results and discussion

We first examined participants' pretest distance estimation scores relative to the actual distance of the target. For these analyses, we used participants' baseline walking speed to convert their imagined walking times into distances. Mean estimated pretest distances for participants were $5.93 \mathrm{~m}$ $(S D=1.67)$ for the $6-\mathrm{m}$ distance, $8.46 \mathrm{~m}(S D=2.70)$ for the $8-\mathrm{m}$ distance, and $11.13 \mathrm{~m}(S D=3.64)$ for the $10-\mathrm{m}$ distance. One-sample $t$-tests comparing each mean with the actual distances revealed that none of these distance estimations differed significantly from the actual target distance.

The goal of Experiment 2a was to examine recalibration of perception and action, this time with an imagined walking distance estimation task at pre- and posttest. A 2 (condition) $\times$ 3 (distance) mixed model ANOVA on percentage scores revealed a significant effect of condition, $F(1,25)=8.54$, $p<.01, \eta_{\mathrm{p}}{ }^{2}=.43$. The mean percentage score in the fast visual 
motion condition $(M=88.2 \%, S D=11.4)$ was significantly smaller than the mean percentage score in the slow visual motion condition $(M=101.5 \%, S D=12.2$; Fig. 4). There was no significant main effect of distance, $F(1,25)=$ $3.76, p=.06$, and no distance $\times$ condition interaction. $F(1,25)=0.01$, n.s.

We also directly compared the imagined distance walked during pretest and posttest in the fast and slow visual motion conditions. Paired samples $t$-tests comparing the average imagined distance walked at pretest with the average imagined distance walked at posttest showed a significant difference between pre- and posttest for the fast condition $(M=$ $6.44 \mathrm{~m}, S D=2.78$ for pretest; $M=5.45 \mathrm{~m}, S D=1.58$ for posttest), $t(13)=2.60, p<.05, d=0.19$, but no significant difference between pre-and posttest for the slow condition $(M=5.83 \mathrm{~m}, S D=1.96$ for pretest; $M=5.84 \mathrm{~m}, S D=1.93$ for posttest), $t(12)=-0.058, p=.955$, n.s.

The results of Experiment $2 \mathrm{a}$ indicate that when the rate of visual motion was manipulated during adaptation, people exhibited a recalibration effect during imagined walking similar to that observed during blindfolded walking (although the effect was stronger for undershooting than for overshooting). The goal of Experiment $2 b$ was to examine whether participants also recalibrate perception and action during imagined walking when the walking speed is manipulated during adaptation while rate of visual motion is held constant.

\section{Experiment 2b: Method}

\section{Participants}

Thirty-four participants (18 females) participated. Participants were recruited in the same manner as in the previous experiments.

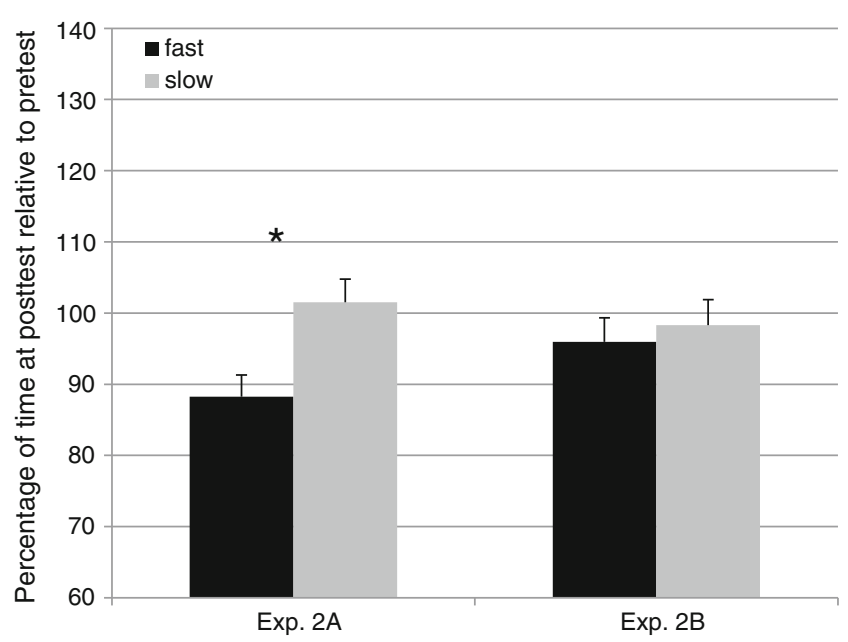

Fig. 4 Percentage of time estimated at posttest, relative to pretest, in fast and slow conditions for Experiments $2 \mathrm{a}$ and $2 \mathrm{~b}$

\section{Apparatus and materials}

The apparatus and materials were identical to those used in Experiment $2 \mathrm{a}$.

\section{Design and procedure}

The imagined walking distance estimation task at pretest and posttest was identical to that used in Experiment 2a. The adaptation phase began immediately after the pretest and was identical to the adaptation phase of Experiment $1 \mathrm{~b}$.

\section{Measures}

Scores represented the percentage of imagined distance walked at posttest relative to pretest. Four participants with a score 1.5 standard deviations above or below the mean (averaged across distances) were excluded from the analyses ( 2 from the fast walking speed condition and 2 from the slow walking speed condition).

\section{Experiment 2b: Results and discussion}

We first examined participants' pretest distance estimation scores relative to the actual target distances. Participants' baseline walking speed was used to convert their imagined walking timed estimates into distances. Mean estimated pretest distances for participants were $5.27 \mathrm{~m}(S D=1.66)$ for the $6-\mathrm{m}$ distance, $7.59 \mathrm{~m}(S D=2.45)$ for the $8-\mathrm{m}$ distance, and $10.00 \mathrm{~m}(S D=3.26)$ for the $10-\mathrm{m}$ distance. One-sample $t$-tests showed that only the 6-m pretest estimate differed significantly from the actual distance, $t(29)=-2.4$, $p<.05$.

The goal of Experiment $2 \mathrm{~b}$ was to test perception-action recalibration using an imagined walking task when action (walking speed) was altered during adaptation, rather than perception (rate of visual motion). A 2 (condition) $\times 3$ (distance) mixed model ANOVA on percentage scores revealed no significant difference between the fast-walking $(M=95.97 \%, S D=12.59)$ and slow-walking $(M=98.3 \%$, $S D=13.95)$ groups, $F(1,28)=0.23$, n.s. (Fig. 4). There was no effect of distance, $F(1,28)=0.02$, n.s., and no distance $\times$ condition interaction, $F(1,28)=0.15$, n.s. Likewise, paired samples $t$-tests comparing the average distance estimated at pretest with the average distance estimated at posttest showed no significant difference between pre- and posttest for the fast condition $(M=5.21 \mathrm{~m}, S D=1.59$ for pretest; $M=4.87 \mathrm{~m}, S D=1.38$ for posttest), $t(14)=1.67, p=.117$, n.s., or the slow condition $(M=5.81 \mathrm{~m}, S D=2.12$ for pretest; $M=5.75 \mathrm{~m}, S D=2.32$ for posttest), $t(14)=0.338$, $p=.740$, n.s.

Although we observed perception-action recalibration when we manipulated rate of visual motion during 
adaptation and used the imagined walking task at test (Experiment 2a), we did not see the recalibration effect when we manipulated the walking speed during adaptation (Experiment 2b). This pattern of results is considered further in the General discussion section.

\section{Experiments 3a and 3b}

Experiments $3 \mathrm{a}$ and $3 \mathrm{~b}$ explored how the testing environment affects the recalibration of action and perception. Of particular interest was whether perception-action coupling is more malleable in a virtual environment. Participants completed both the pre- and posttest imagined walking distance estimation task in the same virtual hallway environment as was used during the adaptation phase. We manipulated rate of visual motion in Experiment $3 \mathrm{a}$ and walking speed in Experiment $3 b$.

\section{Experiment 3a: Method}

\section{Participants}

Thirty-one adults (18 females) participated. Participants were recruited in the same manner as in the previous experiments.

\section{Apparatus and materials}

The apparatus and materials were the same as those used in Experiment 2a. The target consisted of a virtual green circle (13 in. in diameter, 0.25 in. thick) that appeared on the floor of the virtual hallway, with the center of the circle at the target distance for each trial.

\section{Design and procedure}

The pretest used in Experiment 3a was identical to the imagined walking distance estimation pretest used in the previous experiments, except that participants made distance estimations in the virtual hallway instead of the real hallway. After outfitting participants with the safety harness, we first obtained an average walking speed (sans blindfold) for participants by having them walk two lengths of the hallway on the treadmill in the virtual environment. Participants were able to adjust their walking speed to a comfortable rate on the treadmill. The average walking speed on the treadmill for participants in Experiment 3 was $2.91 \mathrm{~m} / \mathrm{s}(S D=0.184)$. During pretest and posttest, participants stood on a $3.81-\mathrm{cm}$-high platform that sat over the treadmill belt so that they would not be standing blindfolded on a treadmill. (When stopped, the treadmill belt tended to move slightly sometimes, which was problematic when participants were standing blindfolded on the treadmill.) For each trial during the distance estimation task, participants viewed a target on the floor of the virtual hallway for $5 \mathrm{~s}$ and then put on a blindfold and imagined walking to the target location. As in the previous experiments, participants completed the two practice trials and nine test trials without feedback.

The adaptation experience was identical to that used in the previous experiments involving manipulation of visual motion. The posttest began immediately after adaptation and was identical to the pretest.

\section{Measures}

Scores represented the percentage of the time estimated at posttest relative to pretest. Five participants with a score 1.5 standard deviations above or below the mean (averaged across distances) were excluded from the analyses (3 from the fast visual motion condition and 2 from the slow visual motion condition).

\section{Experiment 3a: Results and discussion}

We first examined participants' pretest distance estimates relative to the actual target distances. We used participants' treadmill walking speed to convert their estimated times into distances. As in other studies, participants significantly underestimated distances in the virtual environment (Grechkin et al., 2010; Swan et al., 2007; Thompson et al., 2004; Witmer \& Kline, 1998; Witmer \& Sadowski, 1998). Mean estimated pretest distances were $3.66 \mathrm{~m}(S D=1.16)$ for the $6-\mathrm{m}$ distance, $5.62 \mathrm{~m}(S D=1.55)$ for the $8-\mathrm{m}$ distance, and $7.43 \mathrm{~m}(S D=2.14)$ for the $10-\mathrm{m}$ distance. One-sample $t$-tests revealed that all three pretest target distance estimations differed significantly from the actual target distances, $t \mathrm{~s}(25)>-6.1, p<.001$.

The goal of Experiment 3a was to see whether the environment in which participants completed the imagined walking distance estimates affected their recalibration of perception and action. A 2 (condition) $\times 3$ (distance) mixed model ANOVA on percentage scores revealed a highly significant effect of condition, $F(1,24)=35.21, p<.001$, $\eta_{\mathrm{p}}{ }^{2}=.59$. Percentage scores in the fast visual motion condition $(M=77.9 \%, S D=11.13)$ were significantly smaller than percentage scores in the slow visual motion condition $(M=128.99 \%, S D=29.97$; Fig. 5). The underestimation of distance at posttest, relative to pretest, in the fast visual motion condition is particularly remarkable, considering that the pretest estimates were very low to begin with. There was also a significant effect of distance, $F(1,24)=$ 9.07, $p<.01, \eta_{\mathrm{p}}{ }^{2}=.27$, but no significant distance $\times$ condition interaction, $F(1,24)=0.54$, n.s. Mean percentage scores were $108.67 \%(S D=40.35)$ for the $6-\mathrm{m}$ distance, 


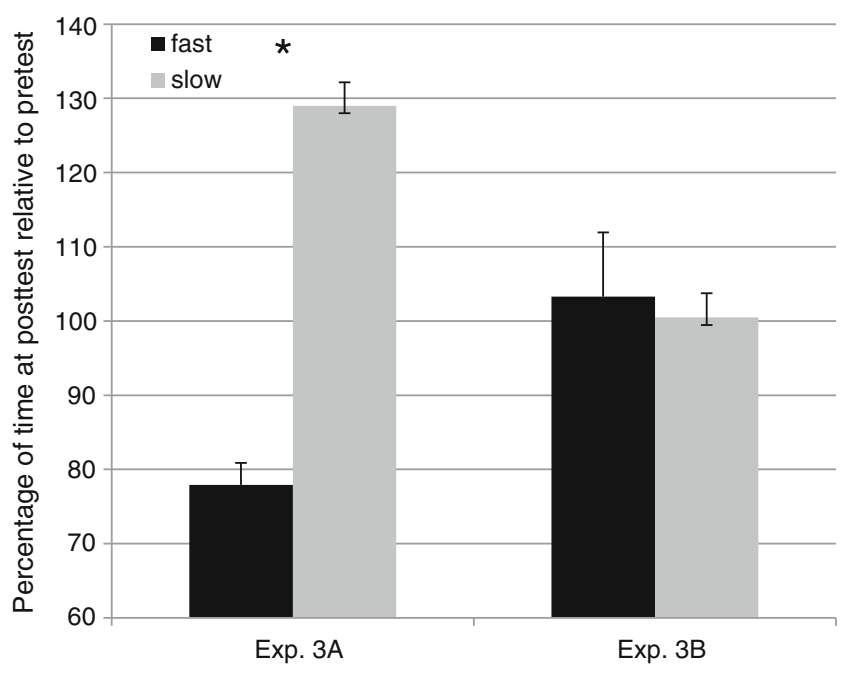

Fig. 5 Percentage of time estimated at posttest, relative to pretest, in fast and slow conditions for Experiments $3 \mathrm{a}$ and $3 \mathrm{~b}$

$96.88 \%(S D=30.70)$ for the $8-\mathrm{m}$ distance, and $98.92 \%$ $(S D=33.44)$ for the $10-\mathrm{m}$ distance. There was a significant difference between 6- and 8-m distances and between the 6and $10-\mathrm{m}$ distances, but not between the 8 and 10-m distances. In addition, paired samples $t$-tests comparing the average distance estimated at pretest versus posttest showed a significant difference between pre- and posttest for both the fast condition $(M=4.24 \mathrm{~m}, S D=1.28$ for pretest; $M=3.21 \mathrm{~m}, S D=.94$ for posttest), $t(13)=7.181, p<.001, d=0.82$, and the slow condition $(M=4.33 \mathrm{~m}, S D=1.14$ for pretest; $M=5.61 \mathrm{~m}$, $S D=2.17$ for posttest), $t(11)=-3.228, p<.01, d=0.43$.

Altering the rate of visual motion while keeping the walking speed constant during the adaptation phase resulted in a larger recalibration effect when participants completed the pre- and posttest distance estimations in the virtual environment, as compared with when they completed preand posttest in the real environment. In Experiment 3b, we tested whether altering the walking speed while holding rate of visual motion constant during adaptation also leads to a strong recalibration effect when participants complete the pre- and posttest in the virtual environment. Although we did not see an effect of recalibration when testing distance estimation via an imagined walking task in the real world (Experiment 2b), we suspected that the less-grounded experience of judging distances in the virtual environment might result in a stronger recalibration effect.

\section{Experiment 3b: Method}

\section{Participants}

Thirty-four adults (15 females) participated in Experiment $3 b$. Participants were recruited in the same manner as in the previous experiments.

\section{Apparatus and materials}

The apparatus and materials were the same as those used in Experiment $3 \mathrm{a}$.

\section{Design and procedure}

The pretest and posttest were identical to those used in Experiment 3a. The adaptation phase followed the same procedure as that in Experiments $1 \mathrm{~b}$ and $2 \mathrm{~b}$.

\section{Measures}

Scores represented the percentage of the time estimated at posttest relative to pretest. Seven participants with a score 1.5 standard deviations above or below the mean (averaged across distances) were excluded from the analyses (4 from the fast walking speed condition and 3 from the slow walking speed condition).

\section{Experiment 3b: Results and discussion}

We did not analyze pretest scores in Experiment 3b, since participants all walked at a predetermined rate on the treadmill and we were unable to use a baseline walking speed to convert imagined walking times into distances.

The goal of Experiment $3 b$ was to determine whether people also exhibited recalibration in an imagined walking task when action, rather than perception, was altered between the two conditions during adaptation. A 2 (condition) $\times 3$ (distance) mixed model ANOVA on percentage scores revealed no significant difference between the fast- and slowwalking groups, $F(1,25)=0.38$, n.s. (Fig. 5). There was a significant effect of distance, $F(1,25)=5.32, p<.05, \eta_{\mathrm{p}}{ }^{2}=$ .18 , but no significant distance $\times$ condition interaction, $F(1,25)=0.31$, n.s. Mean percentage scores were $109.92 \%$ $(S D=24.48)$ for the 6-m distance, $97.21 \%(S D=11.74)$ for the $8-\mathrm{m}$ distance, and $98.37 \%(S D=12.98)$ for the $10-\mathrm{m}$ distance. The score for the 6-m distance was significantly greater than those for the 8-and 10-m distances. There was no significant difference between the 8- and 10-m distances. Thus, when we manipulated walking speed, participants did not exhibit a significant recalibration effect in the imagined walking task even when the pre- and posttest occurred in a virtual environment. In addition, paired samples $t$-tests comparing the average distance estimated at pretest with the average distance estimated at posttest did not show a significant difference between pre- and posttest in either the fast condition $(M=5.38 \mathrm{~m}, S D=1.79$ for pretest; $M=5.49 \mathrm{~m}$, $S D=1.93$ for posttest), $t(12)=-0.541, p=.599$, n.s., or the slow condition $(M=5.35 \mathrm{~m}, S D=1.77$ for pretest; $M=$ $5.25 \mathrm{~m}, S D=1.81$ for posttest), $t(13)=0.813, p=.431$, n.s. 
Recalibration scores in Experiments $2 \mathrm{a}$ and $3 \mathrm{a}$ can be directly compared with those in Experiments $2 b$ and $3 b$ to examine the effect of the environment (real or virtual) in which distance estimates take place. All four of these experiments used imagined walking to test for recalibration; however, in Experiments 2a and 2b, participants were tested in the real environment, and in Experiments $3 a$ and $3 b$, participants were tested in the virtual environment. When comparing Experiments $2 \mathrm{a}$ and $3 \mathrm{a}$, we found that the mean percentage of the distance estimated at posttest, relative to pretest, in the fast visual motion condition of Experiment $3 \mathrm{a}$ $(77.92 \%)$ was significantly lower than the mean percentage of the distance estimated in the fast visual motion condition of Experiment 2a $(88.24 \%), F(26)=5.86, p<.05, \eta_{\mathrm{p}}{ }^{2}=.18$. Likewise, the mean percentage score in the slow visual motion condition of Experiment 3a (128.99 \%) was significantly higher than the mean percentage score in the slow visual motion condition of Experiment 2a (101.51\%), $F(23)=$ 9.30, $p<.01, \eta_{\mathrm{p}}{ }^{2}=.29$. However, when comparing Experiments $2 \mathrm{~b}$ and $3 \mathrm{~b}$, there were no significant differences across experiments in either the fast walking speed condition, $F(26)=2.582$, n.s., or the slow walking speed condition, $F(27)=0.197$, n.s.

When comparing the results of Experiments 2 and 3, we can clearly see that when the rate of visual motion was manipulated, participants showed a much greater recalibration effect when tested in the less-grounded virtual environment, as opposed to the real environment. However, when the walking speed was manipulated, we did not observe a recalibration effect in either the real environment or the virtual environment, likely due to the interaction of the type of recalibration manipulation and the distance estimation task.

\section{General discussion}

The goal of this investigation was to examine how manipulating perception or action during adaptation influences perception-action recalibration in blindfolded walking and imagined walking tasks. We were also interested in the effect of the testing environment on the recalibration effect. In Experiments 1a and 1b, we observed a recalibration effect in the blindfolded walking task when we manipulated either the rate of visual motion (perception) or the walking speed (action). In Experiments $2 \mathrm{a}$ and $2 \mathrm{~b}$, we observed a recalibration effect in the imagined walking task when we manipulated the rate of visual motion during adaptation, but not when we manipulated the walking speed. Likewise, in Experiments $3 \mathrm{a}$ and $3 \mathrm{~b}$, we found that when participants performed the imagined walking task in a virtual environment, they showed a strong recalibration effect when we manipulated the rate of visual motion, but no recalibration effect when we manipulated the walking speed.
The results of these experiments underscore the importance of considering how spatial-updating processes operate on perception and action during the adaptation phase and on representation and action during the test phase of recalibration experiments. During adaptation, participants walk with their eyes open and learn an altered link between perception and action. During test, participants make distance estimations with their eyes closed and must rely on the link between representation and action. It is important to note that in all of these experiments, participants experienced a mismatch between perception and action during adaptation; either they experienced faster or slower visual motion while walking speed was held constant, or they experienced faster or slower walking speed while rate of visual motion was held constant. At test, all participants made estimates about the same target distances. The only thing that differed was the distance estimation task and the testing environment. Below, we consider how these manipulations during adaptation and test influenced the recalibration effect.

Previous studies have examined perception-action recalibration only by manipulating the rate of visual motion, while holding the walking speed constant during adaptation (e.g., Mohler et al., 2004; Mohler et al., 2007; Rieser et al., 1995). Here, we demonstrate that people exhibit recalibration in the blindfolded walking task regardless of whether perception or action is manipulated during adaptation. Specifically, we found significant recalibration when manipulating either rate of visual motion (while holding the walking speed constant) or walking speed (while holding rate of visual motion constant) during adaptation. These results indicate that the ratio of walking speed to visual motion is the critical variable in perception-action recalibration. Through experience interacting with the world, people learn to expect a certain visual gain, relative to movement produced. If this link is altered by manipulating either perception or action, people adapt relatively quickly to this change, as seen when walking blindfolded to targets.

This work also revealed interesting interactions between the type of perception-action manipulation during adaptation and the task used to measure distance estimates. In Experiments $2 \mathrm{a}$ and $2 \mathrm{~b}$, we used the imagined walking task to look at the same change in the perception-action link as we did in Experiments 1a and 1b. Unlike in the blindfolded walking task, we observed recalibration at test when rate of visual motion was manipulated during adaptation, but not when the walking speed was manipulated during adaptation. An important question these findings raise is why do people exhibit perception-action recalibration in imagined walking tasks when the rate of visual motion is manipulated during adaptation, but not when the walking speed is manipulated during adaptation. As was noted earlier, participants in both sets of experiments had exactly the same experiences during 
adaptation. Therefore, the fact that we see recalibration with the blindfolded walking task in both the visual motion and walking speed conditions indicates that participants are, in fact, recalibrating perception and action during the adaptation phase.

One possible reason why we do not see the recalibration effect in the imagined walking task when manipulating the walking speed during adaptation is that participants are actually imagining themselves walking at a faster or slower rate during posttest. Note that we do see differences in walking speed at test when using a blindfolded walking task. Specifically, participants tended to walk significantly faster or slower during posttest in Experiment 1b, while still walking for the same amount of time. It is possible that participants in the fast-walking condition of Experiments $2 \mathrm{~b}$ and $3 \mathrm{~b}$ imagined themselves walking faster and participants in the slow-walking condition imagined themselves walking slower to cover the same amount of distance. If this was the case, participants would have produced very similar time estimates even though they were imagining themselves walking at different rates. This explanation is consistent with a motor simulation account of imagined walking outlined by Kunz et al. (2009). Unfortunately, the imagined walking task has no way of measuring imagined walking speed. In the future, we may be able to further explore this issue by using a continuous pointing method developed by Siegle, Campos, Mohler, Lomis, and Bulthoff (2009). In this method, participants attempt to point continuously at a target off to the side as they imagine themselves moving past it. Methods such as these could help us measure how fast participants are imagining themselves walking during posttest distance estimation and may reveal a difference in walking speed between conditions.

These results also raise the issue of the role of time in the recalibration of perception and action. People may rely on a sense of the amount of time it should take to travel a given distance in order to complete these distance estimation tasks. Typically, researchers emphasize the relationship between speed and distance in recalibrating perception and action. However, it is impossible to separate time out of any equation involving speed and distance, since normally, the amount of time it takes someone to travel a given distance will change along with any change of speed. Therefore, it is important to consider time as a factor in recalibration studies and to consider the role it may play in combination with the distance estimation task used to measure recalibration.

Experiments $3 \mathrm{a}$ and $3 \mathrm{~b}$ examined the role of the test environment on recalibration. We found that when we manipulated the rate of visual motion and tested participants in the virtual environment, participants showed a much stronger recalibration effect than they did when tested in the real environment. Again, participants showed no recalibration effect in the virtual environment when we manipulated walking speed and tested via imagined walking. The fact that participants undershot the distances even more at posttest than at pretest when the rate of visual motion was manipulated is surprising, given how much participants underestimated during pretest in the virtual environment. Why might this be the case? One possibility is that recalibration effects are stronger without the experience of walking blindfolded from the virtual environment into the real environment. However, it seems unlikely that such a large effect as was found in Experiment 3a could be due to this difference alone. On the basis of previous literature, we know that people are less grounded in virtual environments, as compared with the real environment, and tend to underestimate distances until they have been given more experience in the virtual environment (Richardson \& Waller, 2007). Being less grounded may have led participants to rely more heavily on the rate of visual motion information learned during adaptation when completing the imagined walking task in the virtual environment during posttest. In sum, people may rely more heavily on a newly learned perception-action link when the environment is unfamiliar or cue impoverished and they are less grounded in realworld experience. More generally, these results underscore the idea that although virtual environments can serve as useful tools for manipulating perceptual-motor experiences, perception in virtual environments can vary substantially from that in the real environment (see also Nyguyen et al., 2011).

A final issue concerns asymmetries in the degree of overshooting or undershooting in the fast and slow conditions. In the blindfolded walking experiments, participants showed stronger change from pre- to posttest in the slow than in the fast visual motion condition (1a) and in the fastthan in the slow-walking condition (1b). This is consistent with previous work showing that it is more difficult to obtain a recalibration effect in fast than slow visual motion conditions after recalibration in a virtual environment (Mohler et al., 2004; Mohler et al., 2007). In the imagined walking experiments, participants showed stronger change from pre- to posttest in the fast than in the slow visual motion condition in the real environment. This pattern was reversed when the pre- and posttests were conducted in the virtual environment.

How might we explain these asymmetries across the fast and slow conditions? One possibility is that these asymmetries simply reflect random variation across experiments. Another possibility is that task order impacts distance estimates at preand posttest. Given that the posttest is always done second, participants necessarily have greater familiarity with the task at posttest. Greater familiarity with either blindfolded walking or imagined walking to targets could mitigate any effects of the recalibration experience. A third explanation is that experience in a virtual environment impacts distance estimates in 
the real world. Our previous work has shown that imagined walking distance estimates in the real environment are significantly less accurate after experience in a virtual environment than after experience in the real environment (Ziemer et al., 2009). Experience in a virtual environment may also interact with the distance estimation task to produce different patterns of asymmetries between fast and slow conditions. Another explanation is that the asymmetries in the blindfolded walking experiments (1a and $1 \mathrm{~b}$ ) may also be a product of distance compression in virtual environments. Previous research indicates that people tend to underestimate distances in virtual environments, as compared with the real world (e.g., Nguyen et al., 2011; Swan et al., 2007; Thompson et al., 2004; Witmer \& Kline, 1998; Witmer \& Sadowski, 1998). If participants perceived distances as closer during adaptation, the fast visual motion and slow walking speed conditions would seem to correspond more closely to how participants perceived the distances (i.e., less time and effort to reach a given distance). In contrast, the slow visual motion and fast walking speed conditions would seem like a larger divergence from how participants perceived the distances during adaptation, which may explain the larger recalibration effect in these conditions. This account is consistent with the results of Experiment 3 as well; however, it does not explain the larger recalibration effect in the fast visual flow imagined walking experiments in the real world (Experiment 2a). Further work is needed to determine how blindfolded walking and imagined walking distance estimates are impacted by experience in a virtual environment independently of a recalibration experience (i.e., a 1:1 ratio of visual motion to walking speed).

Together, these experiments shed light on the underlying spatial-updating processes used in recalibration tasks. All participants in these six experiments experienced a similar adaptation phase involving a mismatch between the walking speed and rate of visual motion. The experiments with blindfolded walking indicate that the perceptual-motor system is sensitive to the relationship between perception and action, regardless of whether this relationship is altered by manipulating perception or action. The experiments with imagined walking suggest that the spatial-updating processes operating on representation and action are similar across blindfolded and imagined walking. However, further work is needed to better understand imagined motor movement, particularly with respect to the speed of imagined walking.

\section{References}

Decety, J., Jeannerod, M., \& Prablanc, C. (1989). The timing of mentally represented actions. Behavioural Brain Research, 31, $1-5$.

Durgin, F. H., Pelah, A., Fox, L. F., Lewis, J., Kane, R., \& Walley, K. A. (2005). Self-motion perception during locomotor recalibration:
More than meets the eye. Journal of Experimental Psychology. Human Perception and Performance, 31, 398-419.

Farrell, M. J., \& Thomson, J. A. (1998). Automatic spatial updating during locomotion without vision. The Quarterly Journal of Experimental Psychology, 51A, 637-654.

Farrell, M. J., \& Thomson, J. A. (1999). On-line updating of spatial information during locomotion without vision. Journal of Motor Behavior, 31, 39-53.

Grechkin, T. Y., Nguyen, T. D., Plumert, J. M., Cremer, J. F., \& Kearney, J. K. (2010). How does presentation method and measurement protocol affect distance estimation in real and virtual environments? ACM Transactions on Applied Perception, 7, 118.

Interrante, V., Anderson, L., \& Ries, B. (2006). Distance perception in immersive virtual environments, revisited. Proceedings of the IEEE Conference on Virtual Reality, 3-10.

Kunz, B. R., Creem-Regehr, S. H., \& Thompson, W. B. (2009). Evidence for motor simulation in imagined walking. Journal of Experimental Psychology. Human Perception and Performance, $35,1458-1471$

Loomis, J. M., Da Silva, J. A., Fujita, N., \& Fukusima, S. S. (1992). Visual space perception and visually directed action. Journal of Experimental Psychology, 18, 906-921.

Mohler, B. J., Thompson, W. B., Creem-Regehr, S., Pick, H. L., Jr., Warren, W., Jr., Rieser, J. J., \& Willemsen, P. (2004). Visual motion influences locomotion in a treadmill virtual environment. Association for Computing Machinery, Inc., 19-22.

Mohler, B. J., Thompson, W. B., Creem-Regehr, S. H., Willemsen, P., Pick, H. L., Jr., \& Rieser, J. J. (2007). Calibration of locomotion resulting from visual motion in a treadmill-based virtual environment. ACM Transactions on Applied Perception, 4, 1-15.

Nguyen, T. D., Ziemer, C. J., Grechkin, T., Chihak, B., Plumert, J. M., Cremer, J. F., \& Kearney, J. K. (2011). Effects of scale change on distance perception in virtual environments. ACM Transactions on Applied Perception, 8, 1-18.

Plumert, J. M., Kearney, J. K., Cremer, J. F., \& Recker, K. M. (2005). Distance perception in real and virtual environments. $A C M$ Transactions on Applied Perception, 2, 216-233.

Proffitt, D. R., Stefanucci, J., Banton, T., \& Epstein, W. (2003). The role of effort in perceiving distance. Psychological Science, 14, $106-112$.

Richardson, A. R., \& Waller, D. (2007). Interaction with an immersive virtual environment corrects users' distance estimates. Human Factors: The Journal of Human Factors and Ergonomics Society, 49, 507-517.

Rieser, J. J., Ashmead, D. H., Talor, C. R., \& Youngquist, G. A. (1990). Visual perception and the guidance of locomotion without vision to previously seen targets. Perception, 19, 675-689.

Rieser, J. J., Guth, D. A., \& Hill, E. W. (1986). Sensitivity to perspective structure while walking without vision. Perception, 15, 173188.

Rieser, J. J., \& Pick, H. J., Jr. (2007). Using locomotion to update spatial orientation: What changes with learning and development? In J. M. Plumert \& J. P. Spencer (Eds.), The emerging spatial mind (pp. 77-103). New York: Oxford University Press.

Rieser, J. J., Pick, H. J., Jr., Ashmead, D. H., \& Garing, A. E. (1995). Calibration of human locomotion and models of perceptual-motor organization. Journal of Experimental Psychology, 21, 480497.

Sielge, J. H., Campos, J. L., Mohler, B. J., Loomis, J. M., \& Bulthoff, H. H. (2009). Measurement of instantaneous perceived self-motion using continuous pointing. Experimental Brain Research, 195, 429-444.

Swan, J. E. I. I., Jones, A., Kolstad, E., Livingston, M. A., \& Smallman, H. S. (2007). Egocentric depth judgments in optical, 
see-through augmented reality. IEEE Transactions on Visualization and Computer Graphics, 13, 429-442.

Thompson, W. B., Willemsen, P., Gooch, A. A., Creem-Regehr, S. H., Loomis, J. M., \& Beall, A. C. (2004). Does the quality of the computer graphics matter when judging distances in visually immersive environments? Presence: Teleoperators and Virtual Environments, 13, 560-571.

Waller, D., \& Richardson, A. R. (2008). Correcting distance estimates by interacting with immersive virtual environments: Effects of task and available sensory information. Journal of Experimental Psychology. Applied, 14, 61-72.
Withagen, R., \& Michaels, C. F. (2002). The calibration of walking transfers to crawling: Are action systems calibrated? Ecological Psychology, 14, 223-234.

Witmer, B. G., \& Kline, P. B. (1998). Judging perceived and traversed distance in virtual environments. Presence, 7, 144-167.

Witmer, B. G., \& Sadowski, W. J. (1998). Nonvisually guided locomotion to a previously viewed target in real and virtual environments. Human Factors, 40, 478-488.

Ziemer, C. J., Plumert, J. M., Cremer, J. F., \& Kearney, J. K. (2009). Estimating distance in real and virtual environments: Does order make a difference? Attention, Perception, \& Psychophysics, 71, 1095-1106. 